\title{
System-Level Performance of Antenna Arrays in CDMA-Based Cellular Mobile Radio Systems
}

\author{
Andreas Czylwik \\ Department of Communication Systems, University Duisburg-Essen, 47057 Duisburg, Germany \\ Email:czylwik@sent5.uni-duisburg.de
}

Armin Dekorsy

Lucent Technologies GmbH, Bell Labs Innovations, 90411 Nuremberg, Germany

Email: dekorsy@lucent.com

Received 23 June 2003; Revised 1 March 2004

\begin{abstract}
Smart antennas exploit the inherent spatial diversity of the mobile radio channel, provide an antenna gain, and also enable spatial interference suppression leading to reduced intracell as well as intercell interference. Especially, for the downlink of future CDMAbased mobile communications systems, transmit beamforming is seen as a well-promising smart antenna technique. The main objective of this paper is to study the performance of diverse antenna array topologies when applied for transmit beamforming in the downlink of CDMA-based networks. In this paper, we focus on uniform linear array (ULA) and uniform circular array (UCA) topologies. For the ULA, we consider three-sector base stations with one linear array per sector. While recent research on downlink beamforming is often restricted to one single cell, this study takes into account the important impact of intercell interference on the performance by evaluating complete networks. Especially, from the operator perspective, system capacity and system coverage are very essential parameters of a cellular system so that there is a clear necessity of intensive system level investigations. Apart from delivering assessments on the performance of the diverse antenna array topologies, in the paper also different antenna array parameters, such as element spacing and beamwidth of the sector antennas, are optimized. Although we focus on the network level, fast channel fluctuations are taken into account by including them analytically into the signal-to-interference calculation.
\end{abstract}

Keywords and phrases: cellular system, system level simulation, beamforming, uniform linear array, uniform circular array, sectorized system.

\section{INTRODUCTION}

Mobile radio communication represents a rapidly growing market since the global system for mobile communications (GSM) standard has been established. Since then, third generation mobile radio systems like universal mobile telecommunication system (UMTS) or IMT-2000 have already been standardized $[1,2]$ and fourth generation systems are currently investigated. They will probably employ code division multiple access (CDMA) as a multiple access technique. In this paper, we focus on a CDMA-based system with frequency division duplex (FDD) like W-CDMA. A fundamental limitation on the capacity as well as coverage of CDMAbased mobile communication systems is the mutual interference among simultaneous users.

Smart antennas exploit the inherent spatial diversity of the mobile radio channel, provide an antenna gain, and also enable spatial interference suppression leading to reduced intracell as well as intercell interference. However, the implementation of this advanced technique in a handset is difficult with today's hardware due to its limitations in size, cost, and energy storage capability while it is feasible to adopt antenna arrays at base stations.

In such a setting, transmit beamforming at base stations provides a powerful method for increasing downlink capacity $[3,4,5,6]$. But, full exploitation of the spatial properties of the downlink channel requires meaningful transmit channel information at the base station. Third generation mobile systems are designed only with a low rate feedback information channel [5], hence, we focus in this paper on downlink beamforming strategies which are exclusively based on uplink information. While the instantaneous fading is normally uncorrelated between uplink and downlink, it is known that especially for UMTS, the long-term spatial and fading characteristics of the uplink channel can be used for transmit beamforming.

Recent research on downlink beamforming is either restricted on the direct link between a base station and mobile station or by considering only one single cell with few mobile stations. However, it is well known that especially for 
the downlink, the impact of intercell interference on overall system performance plays an important role in CDMAbased systems $[5,7]$. Thus, detailed investigations of downlink beamforming on the network level are strongly required. Note that especially from the operator perspective, system capacity and system coverage are very essential also enhancing the necessity of detailed system level investigations.

The main objective of this paper is to study the performance of diverse antenna array topologies when applied for transmit beamforming in the downlink of CDMA-based networks. In literature, some performance comparisons of systems with different array topologies can be found $[8,9,10$, $11,12]$, but either no real cellular system is considered or important aspects like downlink transmission, maximum ratio combining at the receivers, or specific array topologies are not taken into account. In order to obtain a clear comparison and work out the performance improvement by transmit beamforming, we study omnidirectional as well as 3sector networks whereby the latter concept represents the today's standard antenna configuration. Apart from delivering assessments on the performance of different antenna array topologies in a cellular network, the paper also evaluates and optimizes different antenna array parameters. Note that for the parameter optimization, again, we take into account network level aspects rather than only being focused on the arrays itself.

Our investigations are based on the evaluation of the signal-to-interference ratio (SIR) after RAKE reception at a mobile station. Although we are merely interested in system level results we include fast (instantaneous) fading properties in our investigations. Fast fading is analytically included in the calculation of the SIR values at the mobile stations. This analytical method is a new approach in the area of system level investigations. The key parameter of our investigations is the outage probability that is based on the calculation of the cumulative distribution function (CDF) of the SIR values. An outage occurs if the SIR of a mobile falls below a required SIR threshold.

Finally, it has to be mentioned that the results are based on a simulative approach. Thus, the propagation model plays an important role. Within this paper, we applied a quite realistic propagation model also taking into account the probabilistic nature of all parameters.

The paper is structured as follows. First, Section 2 introduces the basic signal model. Section 3 describes the main parameters for transmit beamforming and also gives a first insight on how to perform downlink beamforming by utilizing long-term uplink spatial mobile radio channel properties. Section 4 deals with the evaluation of the downlink path pattern which is composed of the beamformed pattern, the element-specific pattern, and the azimuthal power spectrum of the individual propagation paths. The latter results from the fact that each (macro)path consists of a large number of micropaths which cause an angular spread of each individual path. Within Section 4, we also calculate the SIR values. Next, in Section 5, the simulation model and simulation parameters are described. Section 6 shows extensive simulation results, and, finally, Section 7 concludes the paper.

\section{SIGNAL MODEL}

For the purpose of this paper, either a uniform linear array (ULA) or a uniform circular array (UCA) is considered for the base station, where the number of array elements for both array topologies is $M$. Mobile stations use one single antenna for transmission and reception only. For notational clarity, it is assumed that the multipath components of the frequency-selective mobile radio channel can be lumped into spatially or temporally resolvable (macro)paths. The number of resolvable paths is determined by the angular resolution of the antenna array and the angular power distribution of the propagation scenario as well as by the relation of the delay spread to the symbol duration of the signal of interest. It is assumed that the number of resolvable paths is the same for uplink and downlink. Here, the number of resolvable paths between the $k$ th mobile station and the $j$ th base station is denoted by $L_{k, j}$. The total number of users in the entire network is $K$ and the number of base stations is $J$. Throughout the whole paper, uplink parameters and variables will be denoted by " " and correspondingly downlink parameters and variables by " "»".

In the following, we focus on uplink transmission at first. The mobile station $k$ is assigned to the base station $j(k)$. At the receiver, the base stations see a sum of resolvable distorted versions of the transmitted signals $\hat{s}_{k}(t)$ of users $k=0, \ldots, K-1$. The complex baseband representation of the antenna array output signal vector of base station $j$ is given by

$$
\hat{\mathbf{r}}_{j}(t)=\sum_{k=0}^{K-1} \sqrt{\hat{P}_{k}} \sum_{l=0}^{L_{k, j}-1} \hat{\mathbf{h}}_{l, k, j} \hat{s}_{k}\left(t-\hat{\tau}_{l, k, j}\right)+\hat{\mathbf{n}}_{j}(t),
$$

where $\hat{P}_{k}$ is the transmitted power from the $k$ th user and $\hat{\mathbf{h}}_{l, k, j}$ represents the channel vector of length $M$ of path $l$ between user $k$ and base station $j$. It is assumed that the channel is quasi time-invariant within the period of interest. The $k$ th user uplink signal $\hat{s}_{k}(t)$ includes the complete baseband signal processing as channel encoding, data modulation, and spreading in case of CDMA transmission and $\hat{\tau}_{l, k, j}$ is the time delay of the $l$ th path between user $k$ and base station $j$. Finally, $\hat{\mathbf{n}}_{j}(t)$ is a spatially and temporally white Gaussian random process with covariance matrix

$$
\hat{\mathbf{R}}_{\mathrm{N}}=\mathrm{E}\left\{\hat{\mathbf{n}}_{j} \hat{\mathbf{n}}_{j}^{H}\right\}=\hat{\sigma}_{\mathrm{N}}^{2} \mathbf{I} \quad \text { for } j=0, \ldots, J-1,
$$

where $\mathrm{E}\{\cdots\}$ denotes the expectation.

The angular spread of the individual incoming resolvable paths determines the amount of spatial fading seen at an antenna array [4] and the size of the array employed will affect the coherence of the array output signals as well as which detection algorithms are applicable. For the rest of this paper, we assume closely spaced antenna elements yielding highly spatially correlated signals at the array elements. For this case, we can express the channel vector as

$$
\hat{\mathbf{h}}_{l, k, j}=\hat{\alpha}_{l, k, j} \hat{\mathbf{a}}\left(\hat{\theta}_{l, k, j}\right),
$$


where $\hat{\alpha}_{l, k, j}$ is the channel coefficient which is composed of path loss, log-normal shadow fading as well as fast Rayleigh fading. The vector $\hat{\mathbf{a}}\left(\hat{\theta}_{l, k, j}\right)$ denotes the array response or steering vector to a planar wave impinging from an azimuth direction $\hat{\theta}_{l, k, j}$. In our model, we assume that the angles of arrival $\hat{\theta}_{l, k, j}$ with $l=0, \ldots, L_{k, j}-1$ are Laplacian-distributed variables with mean $\theta_{k, j}$, the line-of-sight direction between user $k$ and base station $j[13,14]$.

With the assumption of planar waves and uniformly located array elements, the frequency-dependent array response of a ULA is given by $[13,15,16]$

$$
\mathbf{a}_{\mathrm{L}}(\theta)=\left[1, \mathrm{e}^{-\mathrm{j} 2 \pi(d / \lambda) \sin (\theta)}, \ldots, \mathrm{e}^{-\mathrm{j} 2 \pi(M-1)(d / \lambda) \sin (\theta)}\right]^{T} .
$$

The interelement spacing of the antenna array is $d$, and $\lambda$ represents the wavelength of the impinging wave. For the UCA, we have [15]

$$
\begin{aligned}
& \mathbf{a}_{\mathrm{C}}(\theta) \\
& \quad=\left[1, \mathrm{e}^{-\mathrm{j} 2 \pi(R / \lambda) \cos (\theta-2 \pi / M)}, \ldots, \mathrm{e}^{-\mathrm{j} 2 \pi(R / \lambda) \cos (\theta-2 \pi(M-1) / M)}\right]^{T},
\end{aligned}
$$

where $R$ represents the radius of the array.

In order to form a beam for user $k$ and detect its signal at base station $j(k)$, the received vector signal $\hat{\mathbf{r}}_{j(k)}(t)$ is weighted by the weight vector $\hat{\mathbf{w}}_{k}$,

$$
\hat{y}_{k}(t)=\hat{\mathbf{w}}_{k}^{H} \hat{\mathbf{r}}_{j(k)}(t)
$$

These weights depend on the optimization criterion, for example, maximizing the received signal energy (equivalent to SNR), maximizing the SINR, and minimizing the mean squared error between the received signal and some reference signal to be known at the base station [4].

Equation (6) can be rewritten with (1), (3) and either (4) or (5) to

$$
\begin{aligned}
\hat{y}_{k}(t)= & \sqrt{\hat{P}_{k}} \sum_{l=0}^{L_{k, j(k)}-1} \hat{\alpha}_{l, k, j(k)} \hat{\mathbf{w}}_{k}^{H} \hat{\mathbf{a}}\left(\hat{\theta}_{l, k, j(k)}\right) \hat{s}_{k}\left(t-\hat{\tau}_{l, k, j(k)}\right) \\
& +\sum_{\substack{\kappa=0 \\
\kappa \neq k}}^{K-1}{\sqrt{\hat{P}_{\kappa}}}^{L_{k, j(k)}-1} \sum_{l=0} \hat{\alpha}_{l, \kappa, j(k)} \hat{\mathbf{w}}_{k}^{H} \hat{\mathbf{a}}\left(\hat{\theta}_{l, \kappa, j(k)}\right) \hat{s}_{\kappa}\left(t-\hat{\tau}_{l, \kappa, j(k)}\right) \\
& +\hat{\mathbf{w}}_{k}^{H} \hat{\mathbf{n}}_{j(k)}(t) .
\end{aligned}
$$

The first term describes the desired signal, the second term represents the intercell as well as intracell interference, and the last expression describes additive Gaussian noise. Assuming that the data signals $\hat{s}_{k}\left(t-\hat{\tau}_{l, k, j(k)}\right)$ and the additive noise $\hat{\mathbf{n}}_{j(k)}(t)$ are zero-mean and statistically independent random processes, the total received uplink signal power of the user of interest at the base station can be expressed in the form

$$
\begin{aligned}
\hat{P}_{\mathrm{R}, k}= & \mathrm{E}\left\{\left|\hat{y}_{k}(t)\right|^{2}\right\} \\
= & \hat{P}_{k} \sum_{l=0}^{L_{k, j(k)}-1}\left|\hat{\alpha}_{l, k, j(k)}\right|^{2} \cdot\left|\hat{\mathbf{w}}_{k}^{H} \hat{\mathbf{a}}\left(\hat{\theta}_{l, k, j(k)}\right)\right|^{2} \\
& +\sum_{\substack{\kappa=0 \\
\kappa \neq k}}^{K-1} \hat{P}_{\kappa} \sum_{l=0}^{L_{\kappa, j(k)}-1}\left|\hat{\alpha}_{l, \kappa, j(k)}\right|^{2} \cdot\left|\hat{\mathbf{w}}_{k}^{H} \hat{\mathbf{a}}\left(\hat{\theta}_{l, \kappa, j(k)}\right)\right|^{2} \\
& +\mathrm{E}\left\{\left|\hat{\mathbf{w}}_{k}^{H} \hat{\mathbf{n}}_{j(k)}(t)\right|^{2}\right\} \\
= & \hat{\mathbf{w}}_{k}^{H} \hat{\mathbf{R}}_{\mathrm{S}, k} \hat{\mathbf{w}}_{k}+\hat{\mathbf{w}}_{k}^{H} \hat{\mathbf{R}}_{\mathrm{I}, k} \hat{\mathbf{w}}_{k}+\hat{\mathbf{w}}_{k}^{H} \hat{\mathbf{R}}_{\mathrm{N}} \hat{\mathbf{w}}_{k},
\end{aligned}
$$

where the expectation operation is carried out with respect to the fast varying data signal and the additive noise. Note that the expectation is not carried out with respect to the fast fading processes, since we assume that the channel remains unchanged during a block of data. Here, it has been assumed that also time-delayed versions of the same data signal are uncorrelated. The $k$ th user signal is normalized by $\mathrm{E}\left\{\left|s_{k}\right|^{2}\right\}=1$ for $k=0, \ldots, K-1$. The essential elements in antenna array beamforming design are the spatial covariance matrices $\hat{\mathbf{R}}_{\mathrm{S}, k}$ for the desired signal as well as the spatial covariance matrices $\hat{\mathbf{R}}_{\mathrm{I}, k}$ for the interference of user $k$. Both matrices are instantaneous covariance matrices which are fluctuating according to fast fading. According to (8), these matrices are given by

$$
\begin{aligned}
\hat{\mathbf{R}}_{\mathrm{S}, k} & =\hat{P}_{k} \sum_{l=0}^{L_{k, j(k)}-1}\left|\hat{\alpha}_{l, k, j(k)}\right|^{2} \cdot \hat{\mathbf{a}}\left(\hat{\theta}_{l, k, j(k)}\right) \hat{\mathbf{a}}\left(\hat{\theta}_{l, k, j(k)}\right)^{H}, \\
\hat{\mathbf{R}}_{\mathrm{I}, k} & =\sum_{\substack{\kappa=0 \\
\kappa \neq k}}^{K-1} \hat{P}_{\kappa} \sum_{l=0}^{L_{\kappa, j(k)}-1}\left|\hat{\alpha}_{l, \kappa, j(k)}\right|^{2} \cdot \hat{\mathbf{a}}\left(\hat{\theta}_{l, \kappa, j(k)}\right) \hat{\mathbf{a}}\left(\hat{\theta}_{l, \kappa, j(k)}\right)^{H} .
\end{aligned}
$$

These covariance matrices include all the spatial information necessary for beamforming. They can be measured in the uplink by correlating all antenna array output signals,

$$
\mathrm{E}\left\{\hat{\mathbf{r}}_{j(k)} \hat{\mathbf{r}}_{j(k)}^{H}\right\}=\hat{\mathbf{R}}_{\mathrm{S}, k}+\hat{\mathbf{R}}_{\mathrm{I}, k}+\hat{\mathbf{R}}_{\mathrm{N}} .
$$

The only remaining task is to distinguish between the contribution of the desired signal and the contribution of interference plus noise. This can be accomplished by evaluating user-specific training sequences.

Next, downlink transmission is considered. A mobile terminal receives the desired signal from the base station to which it is connected. But it also receives interference from all other base stations. The received signal is given by

$$
\begin{aligned}
\check{y}_{k}(t)= & \sqrt{\check{P}_{k}} \sum_{l=0}^{L_{k, j(k)}-1} \check{\alpha}_{l, k, j(k)} \check{\mathbf{w}}_{k}^{H} \check{\mathbf{a}}\left(\check{\theta}_{l, k, j(k)}\right) \check{s}_{k}\left(t-\check{\tau}_{l, k, j(k)}\right) \\
& +\check{i}_{k}(t)+\check{n}_{k}(t) .
\end{aligned}
$$

The first term in (12) is the desired signal and the second term $\check{i}_{k}(t)$ is interference which is composed from intracell as well as intercell interference. The last term $\check{n}_{k}(t)$ is additive 
white Gaussian noise which is created from thermal and amplifier noise. Assuming that the data signals for different mobile stations are statistically independent and that also timedelayed versions of the same data signal are uncorrelated, the power of the received signal at mobile station $k$ yields

$$
\begin{aligned}
\check{P}_{\mathrm{R}, k}= & \mathrm{E}\left\{\left|\check{y}_{k}(t)\right|^{2}\right\} \\
= & \check{P}_{k} \sum_{l=0}^{L_{k, j(k)}-1}\left|\check{\alpha}_{l, k, j(k)}\right|^{2} \cdot\left|\check{\mathbf{w}}_{k}^{H} \check{\mathbf{a}}\left(\check{\theta}_{l, k, j(k)}\right)\right|^{2} \\
& +\mathrm{E}\left\{\left|\check{i}_{k}\right|^{2}\right\}+\mathrm{E}\left\{\left|\check{n}_{k}\right|^{2}\right\} \\
= & \check{\mathbf{w}}_{k}^{H} \check{\mathbf{R}}_{\mathrm{S}, k} \check{\mathbf{w}}_{k}+\mathrm{E}\left\{\left|\check{i}_{k}\right|^{2}\right\}+\mathrm{E}\left\{\left|\check{n}_{k}\right|^{2}\right\} .
\end{aligned}
$$

Here, $\check{\mathbf{R}}_{\mathrm{S}, k}$ denotes the downlink covariance matrix for the desired signal component

$$
\check{\mathbf{R}}_{\mathrm{S}, k}=\check{P}_{k} \sum_{l=0}^{L_{k, j(k)}-1}\left|\check{\alpha}_{l, k, j(k)}\right|^{2} \cdot \check{\mathbf{a}}\left(\check{\theta}_{l, k, j(k)}\right) \check{\mathbf{a}}\left(\check{\theta}_{l, k, j(k)}\right)^{H} .
$$

For an FDD system, fast fading processes in uplink and downlink are almost uncorrelated. Therefore, the instantaneous uplink covariance matrix cannot be used directly for downlink beamforming. But on the other hand, measurements have shown that the following spatial transmission characteristics for uplink and downlink are almost the same if the frequency spacing between uplink and downlink bands is not too large (see [17], [18, Section 3.2.2], [19]):

$$
\begin{aligned}
\hat{\theta}_{l, k, j} & \cong \check{\theta}_{l, k, j}, \\
\hat{\tau}_{l, k, j} & \cong \check{\tau}_{l, k, j}, \\
\mathrm{E}\left\{\left|\hat{\alpha}_{l, k, j}\right|^{2}\right\} & \cong \mathrm{E}\left\{\left|\check{\alpha}_{l, k, j}\right|^{2}\right\} .
\end{aligned}
$$

In (17), the expectation is taken over the fast fading processes. The equation implies that fading processes from shadowing are almost the same for uplink and downlink. Because of this reason, a part of the spatial information which is available from the uplink covariance matrices can be utilized also for the downlink.

Since the instantaneous full spatial information is not available for the downlink, downlink beamforming has to be based on averages (with respect to fast fading) of the covariance matrices.

\section{DOWNLINK BEAMFORMING}

The scope of this paper is to investigate different antenna array topologies for downlink beamforming. To fully exploit spatial filtering capabilities, complete downlink spatial information is required at the base station to reduce intercell as well as intracell interference. Complete spatial information comprises the knowledge of the covariance matrices which include the knowledge of instantaneous magnitudes of the channel coefficients $\left|\alpha_{l, k, j(k)}\right|$, the angles of arrival $\theta_{l, k, j(k)}$, and transmitted powers $P_{k}$. The beamforming strategy which will be discussed later in this section is directly based on covariance matrices.
Usually, spatial information is only available for uplink transmission by evaluating user-specific training sequences at base stations. For the downlink, a backward transmission of channel state information from the mobile stations to the base stations would be necessary. Since mobile communication systems are commonly designed with low data rate signalling feedback channels in order to obtain high bandwidth efficiency (e.g., UMTS [5]), neither the instantaneous channel coefficients nor steering vectors are known at the base station. Although the fast fading processes for uplink and downlink are uncorrelated, the averaged (with respect to fast fading) magnitudes of channel coefficients can be assumed to be insensitive to small changes in frequency. Thus, the averaged channel coefficients and angles of arrival can be estimated from the time-averaged uplink covariance matrices. For power control procedures which are controlled by base stations, all transmitted power levels are also known at the base stations.

The following methods can be used to estimate the downlink covariance matrices.

(i) After estimation of angles of arrival and power transfer factors with high resolution estimation methods [20] from the time-averaged uplink covariance matrices, the downlink covariance matrices are calculated using (14).

(ii) Alternatively, the covariance matrices are transformed directly from uplink to downlink carrier frequency by linear transformations as proposed in literature $[21,22,23]$.

(iii) Furthermore, it is possible to feedback the averaged downlink covariance matrix which may be measured at the mobile station. But this concept requires a high data rate feedback channel which allows to feedback the analog values of the elements of the covariance matrix. This concept can also be used for interference, but only within the considered cell-the contribution of intercell interference cannot be taken into account.

Of course, estimation errors cause some degradation compared with the ideal case where the covariance matrices are exactly known. For simplicity and in order to estimate the ultimate performance, in this paper we assume perfectly known time-averaged downlink covariance matrices.

The beamforming strategy in the present paper is to maximize the received signal power at mobile station $k$. The instantaneous received power at mobile station $k$ is given by

$$
\check{P}_{\mathrm{S}, k}=\check{\mathbf{w}}_{k}^{H} \check{\mathbf{R}}_{\mathrm{S}, k} \check{\mathbf{w}}_{k},
$$

where $\check{\mathbf{R}}_{\mathrm{S}, k}$ denotes the instantaneous downlink covariance matrix of the desired signal (14). As mentioned before, the instantaneous downlink covariance matrix is not known at the base station. Instead, we are using the time-averaged version which can be calculated with the above described methods. Therefore, the beamforming algorithm is based on the time-averaged downlink covariance matrix $\tilde{\mathbf{R}}_{\mathrm{S}, k}$ which 
corresponds to the expectation

$$
\begin{aligned}
\tilde{\mathbf{R}}_{\mathrm{S}, k} & =\mathrm{E}\left\{\check{\mathbf{R}}_{\mathrm{S}, k}\right\} \\
& =\check{P}_{k} \sum_{l=0}^{L_{k, j(k)}-1} \mathrm{E}\left\{\left|\check{\alpha}_{l, \kappa, j(k)}\right|^{2}\right\} \cdot \check{\mathbf{a}}\left(\check{\theta}_{l, \kappa, j(k)}\right) \check{\mathbf{a}}\left(\check{\theta}_{l, \kappa, j(k)}\right)^{H} .
\end{aligned}
$$

Be aware that the steering vectors have to be determined at downlink frequency. Because we are averaging with respect to Rayleigh fading, the actual beamforming for the downlink is to maximize the average downlink power

$$
\tilde{P}_{\mathrm{S}, k}=\check{\mathbf{w}}_{k}^{H} \tilde{\mathbf{R}}_{\mathrm{S}, k} \check{\mathbf{w}}_{k},
$$

while keeping the average total intracell and intercell interference power $\tilde{P}_{\mathrm{I}, k}$ transmitted from base station $j(k)$ and received from all undesired mobile stations constant

$$
\begin{aligned}
\tilde{P}_{\mathrm{I}, k} & =\sum_{\substack{\kappa=0 \\
\kappa \neq k}}^{K-1} \mathrm{E}\left\{\left|\check{y}_{\kappa}(t)\right|^{2}\right\} \\
& =\check{P}_{k} \sum_{\substack{\kappa=0 \\
\kappa \neq k}}^{K-1} \sum_{l=0}^{L_{\kappa, j(k)}-1} \mathrm{E}\left\{\left|\check{\alpha}_{l, \kappa, j(k)}\right|^{2}\right\} \cdot\left|\check{\mathbf{w}}_{k}^{H} \mathbf{a}\left(\check{\theta}_{l, \kappa, j(k)}\right)\right|^{2} \\
& =\check{\mathbf{w}}_{k}^{H} \tilde{\mathbf{R}}_{\mathrm{I}, k} \check{\mathbf{w}}_{k} .
\end{aligned}
$$

Here, $\tilde{\mathbf{R}}_{\mathrm{I}, k}$ denotes the downlink interference covariance matrix (averaged with respect to the data signals and Rayleigh fading processes):

$$
\tilde{\mathbf{R}}_{\mathrm{I}, k}=\check{P}_{k} \sum_{\substack{\kappa=0 \\ \kappa \neq k}}^{K-1} \sum_{l=0}^{L_{\kappa, j(k)}-1} \mathrm{E}\left\{\left|\check{\alpha}_{l, \kappa, j(k)}\right|^{2}\right\} \cdot \check{\mathbf{a}}\left(\check{\theta}_{l, \kappa, j(k)}\right) \stackrel{\mathbf{a}}{ }\left(\check{\theta}_{l, \kappa, j(k)}\right)^{H} .
$$

Considering an interference-limited system and therefore neglecting the additive noise powers $\mathrm{E}\left\{\left|\check{n}_{k}(t)\right|^{2}\right\}$, the described beamforming strategy corresponds to maximizing the (virtual) SIR per user, which is given by

$$
\operatorname{SIR}_{k}=\frac{\check{\mathbf{w}}_{k}^{H} \tilde{\mathbf{R}}_{\mathrm{S}, k} \check{\mathbf{w}}_{k}}{\check{\mathbf{w}}_{k}^{H} \tilde{\mathbf{R}}_{\mathrm{I}, k} \check{\mathbf{w}}_{k}} .
$$

Note that the SIR of (23) cannot be measured at any terminal since the denominator contains the sum of interference powers measured at different mobile stations. Therefore, we call it virtual SIR.

The optimization problem to maximize the SIR can mathematically be expressed as

$$
\check{\mathbf{w}}_{k}^{\text {opt }}=\arg \max _{\check{\mathbf{w}}_{k}} \frac{\check{\mathbf{w}}_{k}^{H} \tilde{\mathbf{R}}_{\mathrm{S}, k} \check{\mathbf{w}}_{k}}{\check{\mathbf{w}}_{k}^{H} \tilde{\mathbf{R}}_{\mathrm{I}, k} \check{\mathbf{w}}_{k}},
$$

where $\check{\mathbf{w}}_{k}^{\text {opt }}$ represents the optimum solution. Since both covariance matrices are positive definite, the maximum SIR criterion is satisfied when the weight vector equals the principal eigenvector of the matrix pair associated with the largest eigenvalue $[4,13,21]$, that is,

$$
\tilde{\mathbf{R}}_{\mathrm{S}, k} \check{\mathbf{w}}_{k}^{\text {opt }}=\lambda_{\max } \tilde{\mathbf{R}}_{\mathrm{I}, k} \check{\mathbf{w}}_{k}^{\text {opt }},
$$

where $\lambda_{\max }$ denotes the largest eigenvalue.

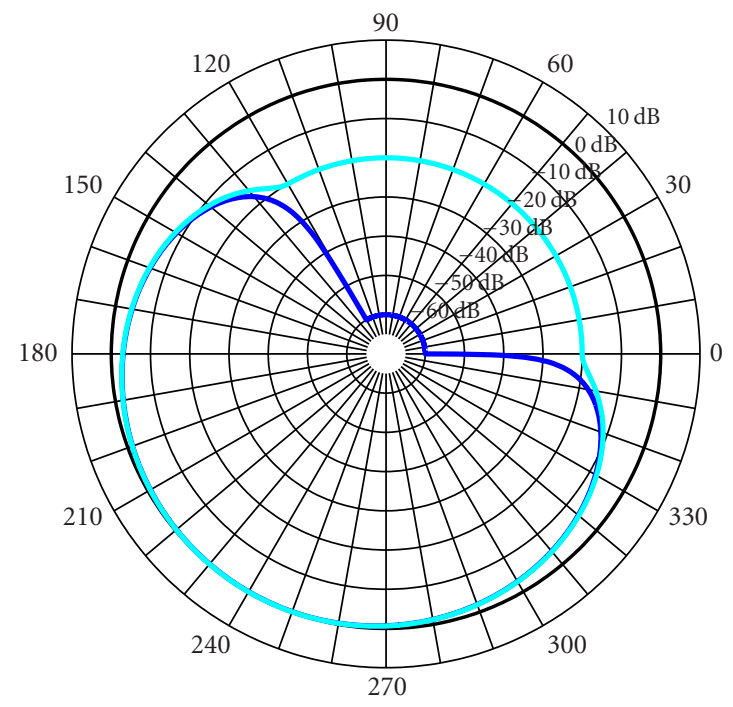

FIGURE 1: Antenna diagram of a single antenna element (main beam direction, $240^{\circ}$ ), backward attenuation $a_{\mathrm{R}}=20 \mathrm{~dB}$ and $a_{\mathrm{R}}=60 \mathrm{~dB}$.

\section{DOWNLINK SIR}

The total gain of the antenna array is given by [15],

$$
\check{G}_{k}^{\text {tot }}(\theta)=\left|\check{\mathbf{w}}_{k}^{\text {opt }} \mathbf{a}(\theta)\right|^{2} \cdot G^{\text {ele }}(\theta),
$$

where the first term is due to the applied beamforming method and dependent on the topology used, $\mathbf{a}(\theta)$ is given by (4) or (5), respectively. The second term takes into account the antenna element specific antenna pattern. Typical patterns of base station sector antennas show a smooth behavior within the main beam. Such a characteristic can be modelled quite well with a squared cosine characteristic. Within this paper, we apply antenna elements with squared cosine shapes in the form

$$
G^{\mathrm{ele}}(\theta)= \begin{cases}\cos ^{2}\left(\frac{\pi}{2} \cdot \frac{\theta}{\theta_{3 \mathrm{~dB}}}\right) & \text { for }|\theta| \leq \theta_{0}, \\ 10^{-a_{\mathrm{R}} / 10} & \text { for }|\theta| \geq \theta_{0},\end{cases}
$$

with $\theta_{0}=\theta_{3 \mathrm{~dB}} \cdot 2 / \pi \cdot \arccos 10^{-a_{\mathrm{R}} / 20}$. In (27), the angle $\theta_{3 \mathrm{~dB}}$ is the $3 \mathrm{~dB}$ two-sided angular aperture of an antenna element (often termed half-power beamwidth) and $a_{\mathrm{R}}$ denotes the backward attenuation. By taking very large values for $\theta_{3 \mathrm{~dB}}$, an omnidirectional antenna characteristic can be modelled. The specific shape of the antenna characteristic plays only a subordinate role as is shown later in this paper. Even if the $3 \mathrm{~dB}$ angular aperture is changed in a large range, no significant performance difference is found. If not otherwise declared, a $3 \mathrm{~dB}$ angular aperture of $120^{\circ}$ is used. Figure 1 illustrates the antenna element-specific diagram. For ULAs, Figure 2 shows the orientation of $120^{\circ}$ sectors in the cellular system and illustrates the sectorization of cells.

As introduced before, each resolvable path at the base station receiver is composed of micropaths (often modelled by many small scatterers) with slightly different angles of arrival at the antenna arrays. Thus, the power is spread around the 


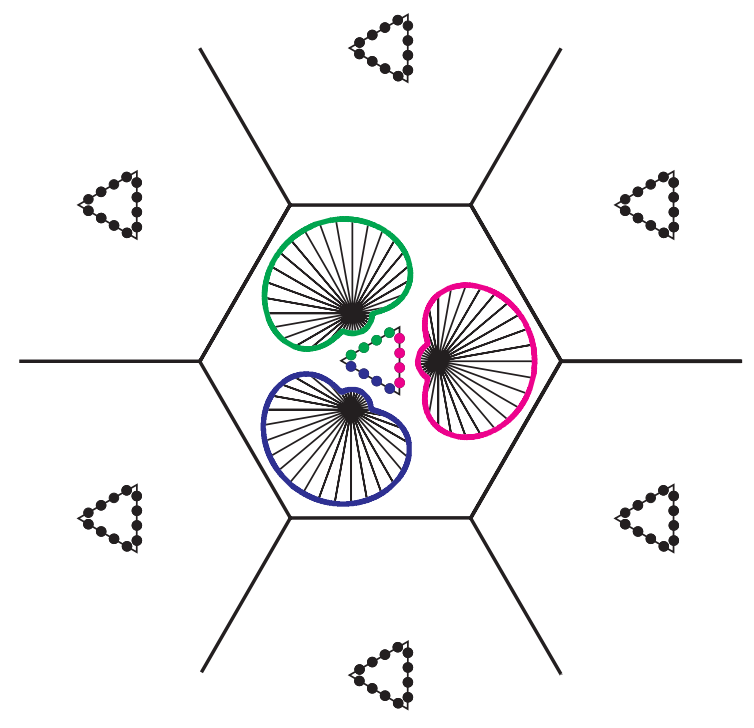

FIgURE 2: Single cell with antenna diagrams of the sector antennas.

average angle of arrival $\check{\theta}_{l, k, j(k)}$ of each resolvable path and a (path-specific) azimuthal power spectrum has to be incorporated in the calculation of the signal and interference power for downlink transmission. To carry out the calculation we again fall back on the long-term reciprocity of the uplink and the downlink channel, refer to (15), (16), and (17). For the rest of this paper, we assume identical Laplacian-shaped azimuthal power spectra $p_{l, k, j}(\theta)=p(\theta)$ for all paths in the system $[13,24]$. With this assumption, the resulting gain factor seen by the $l$ th departing path of user $k$ at base station $j(k)$ can be evaluated by convolving the total antenna gain diagram (26) with the azimuthal power spectrum,

$$
G_{k}^{\text {path }}\left(\check{\theta}_{l, k, j}\right)=\int_{-\pi}^{\pi} \check{G}_{k}^{\text {tot }}(\theta) p\left(\theta-\check{\theta}_{l, k, j}\right) d \theta .
$$

Within this paper, $G_{k}^{\text {path }}$ is also referred to as path diagram [25].

In the following, we will give an expression for the SIR at a mobile station based on beamformed antenna diagrams at all base stations in the network. We consider CDMA systems with RAKE reception and assume the systems to be interference limited. Thus, the influence of thermal and amplifier noise can be neglected. With these assumptions and with reference on (13), the (instantaneous) postdespreading SIR per path of the user of interest (indexed with $k$ ) is given by

$$
\gamma_{l, k}=\frac{G_{S} \check{P}_{l, k}}{\check{P}_{l, k}^{\text {cross }}+\check{P}_{k}^{\text {intra }}+\check{P}_{k}^{\text {inter }}}, \quad l=0, \ldots, L_{k, j(k)}-1,
$$

with path power

$$
\check{P}_{l, k}=\check{P}_{k}\left|\check{\alpha}_{l, k, j(k)}\right|^{2} \check{G}_{k}^{\text {path }}\left(\check{\theta}_{l, k, j(k)}\right)
$$

and path-crosstalk interference [26]

$$
\check{P}_{l, k}^{\text {cross }}=\sum_{\substack{l^{\prime}=0 \\ l^{\prime} \neq l}}^{L_{k, j(k)}-1} \check{P}_{k}\left|\check{\alpha}_{l^{\prime}, k, j(k)}\right|^{2} \check{G}_{k}^{\text {path }}\left(\check{\theta}_{l^{\prime}, k, j(k)}\right) .
$$

Here, $\check{P}_{k}$ with $k=0, \ldots, K-1$ denotes the transmitted power to be adjusted by power control $[27,28,29]$. In the present paper, we neglect the effect of power control and therefore assume $\check{P}_{k}=\stackrel{P}{\text { for }} k=0, \ldots, K-1$. Since we focus on CDMA systems, $G_{S}$ denotes the processing gain (despreading gain) $[5,26]$. The variable $\check{\alpha}_{l, k, j(k)}$ is given by (17) and includes signal fading. In implementable CDMA receivers, the number of paths to be evaluated is determined by the applied number of RAKE fingers [26]. Since we are interested in upper bound assessments for beamforming concepts, we neglect this restriction and assume all paths to be exploited by the RAKE receiver. Note that this leads to the highest degree of achievable path diversity in the time domain [26]. The intracell interference power yields

$$
\check{P}_{k}^{\text {intra }}=\sum_{\kappa \in \mathcal{A}_{k}} \sum_{l=0}^{L_{k, j(k)}-1} \check{P}_{\kappa}\left|\check{\alpha}_{l, k, j(k)}\right|^{2} \check{G}_{\kappa}^{\text {path }}\left(\check{\theta}_{l, k, j(k)}\right) .
$$

The set $\mathcal{A}_{k}$ contains intracell interferers of user $k$. Note that the intracell interference signals pass through the same mobile channel as the signals of the user of interest, but they are weighted with their corresponding user-specific path diagram $\breve{G}_{\kappa}^{\text {path }}$. Finally, the intercell interference power can be expressed as

$$
\check{P}_{k}^{\text {inter }}=\sum_{\kappa \in B_{k}} \sum_{l=0}^{L_{k, j(\kappa)}-1} \check{P}_{\kappa}\left|\check{\alpha}_{l, k, j(\kappa)}\right|^{2} \check{G}_{\kappa}^{\text {path }}\left(\check{\theta}_{l, k, j(\kappa)}\right),
$$

where $\mathcal{B}_{k}, k=0, \ldots, K-1$, describes the set of users causing intercell interference seen by the $k$ th user. The interference signals differ from the signals of interest by the mobile channels as well as path diagrams. Note that a large number of interfering signals arrives at each mobile. Thus, it is valid to approximate the path cross talk interference by including the path of interest, that is, $\check{P}_{l, k}^{\text {cross }} \approx \sum_{l} \check{P}_{k}\left|\check{\alpha}_{l, k, j(k)}\right|^{2} \breve{G}_{k}^{\text {path }}\left(\check{\theta}_{l, k, j(k)}\right)$. This leads to identical interference powers (identical denominators in (29)) for all paths and simplifies the following analysis.

System level simulations often neglect short-term aspects as fast fading. Within this paper, we introduce a new approach which takes fast fading into account. First, it has to be mentioned that combining the resolvable paths is done by maximum ratio combining (MRC). Secondly, rather than explicitly modelling fast fading, we mathematically incorporate it in the evaluation of the SIR distribution when MRC is applied for different path power transfer factors $[24,26]$.

The key parameter of our investigations is the CDF of the SIR. It is assumed that all channel coefficients $\check{\alpha}_{l, k, j}$ are complex Gaussian random variables which correspond to Rayleigh fading magnitudes. We furthermore presume that 
the channel coefficients $\check{\alpha}_{l, k, j}$ are statistically independent. The path gain factor $\breve{G}_{k}^{\text {path }}\left(\check{\theta}_{l, k, j(k)}\right)$ in (30) depends on the optimum beam pattern (solution of (25)) which changes only very slowly with time since it is based on time-averaged covariance matrices. Because of the large number of terms in the denominator of (29), we can neglect the fluctuations of the denominator. Therefore, the only variables which fluctuate because of the Rayleigh fading are the channel coefficients $\check{\alpha}_{l, k, j}$. The Gaussian distribution of channel coefficients results in an exponentially distributed signal power per path (numerator of (29)). Since the interference power and all other terms of (29) (except the coefficients $\check{\alpha}_{l, k, j}$ ) are assumed to be fixed or very slowly fluctuating, the signal-tointerference power ratios $\gamma_{l, k}$ per path are distributed according to an exponential distribution [26], that is,

$$
f_{\gamma_{l, k}}\left(\gamma_{l, k}\right)=\frac{1}{\overline{\gamma_{l, k}}} \mathrm{e}^{-\left(\gamma_{l, k}\right) /\left(\overline{\gamma_{l, k}}\right)},
$$

where $\overline{\gamma_{l, k}}$ denotes the average SIR of a single path (ensemble average with respect to fast fading). Assuming that the interference in each path is independent, the SIR after MRC results in

$$
\gamma_{k}=\sum_{l=0}^{L_{k, j(k)}-1} \gamma_{l, k} .
$$

Furthermore, it is assumed that the small scale fading of the individual desired paths is statistically independent. Since $\gamma_{k}$ is the sum of the random variables $\gamma_{l, k}$, the resulting probability density function (PDF) is obtained from convolving the individual PDFs,

$$
f_{\gamma_{k}}\left(\gamma_{k}\right)=f_{\gamma_{1, k}} * f_{\gamma_{2, k}} * f_{\gamma_{3, k}} * \cdots * f_{\gamma_{L_{k, n}(k)^{-1, k}}} .
$$

Utilizing the characteristic functions of the PDFs, the resulting PDF of $\gamma_{k}$ can be found to be $[24,26]$

$$
f_{\gamma_{k}}\left(\gamma_{k}\right)=\sum_{l=0}^{L_{k, j(k)}-1} \frac{c_{l, k}}{\overline{\gamma_{l, k}}} \mathrm{e}^{-\gamma_{k} / \overline{\gamma_{l, k}}}
$$

with the coefficients

$$
c_{l, k}=\prod_{\substack{l^{\prime}=0 \\ l^{\prime} \neq l}}^{L_{k, j(k)}-1} \frac{\overline{\gamma_{l, k}}}{\overline{\gamma_{l, k}}-\overline{\gamma_{l^{\prime}, k}}} .
$$

In order to compare the different beamforming concepts, the CDF has to be averaged over all mobiles and possibly over several simulations, where different locations for the mobiles and different radio channels are determined. Most information can be extracted from the averaged distribution function of the SIR,

$$
F_{\gamma_{k}}=\int_{0}^{\gamma_{k}} E\left\{f_{\gamma_{k}}(u)\right\} \mathrm{d} u,
$$

where the expectation is taken over all mobile stations and snapshots.

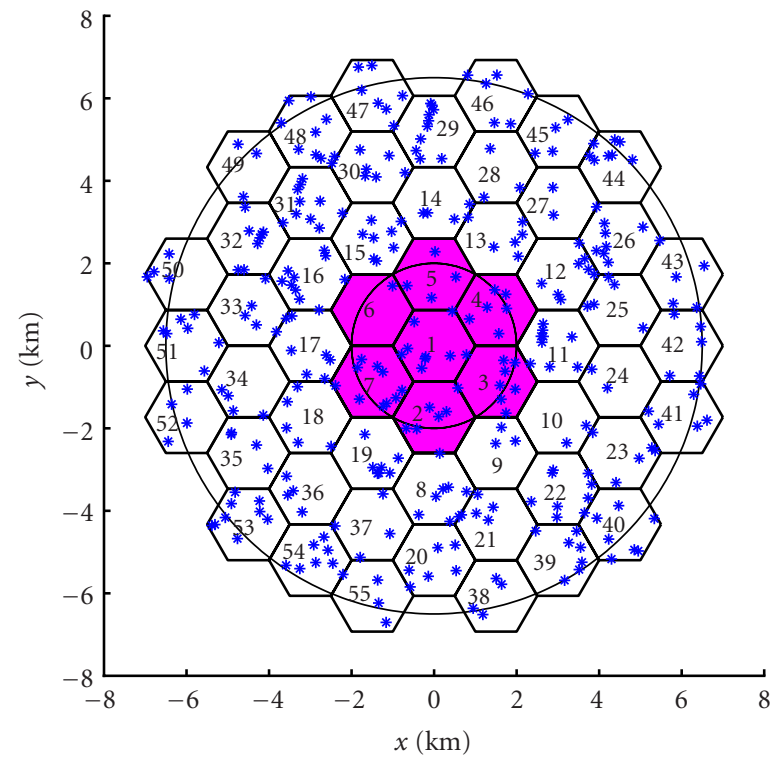

FIGURE 3: Cellular simulation model with reference cells (grey) in the center and randomly distributed mobile stations.

\section{CELLULAR SIMULATION MODEL AND METHODOLOGY}

\subsection{Simulation model}

The simulations are carried out with a regular hexagonal cellular model (see Figure 3). In order to be able to ignore fringe effects, the SIR is calculated only in a central area (reference cells). Mobile stations are randomly distributed in the cellular system according to a spatially uniform distribution. Note that a realistic model of the wave propagation plays an important role for the significance of the simulation results. One common approach, especially in context of downlink beamforming, is to use deterministic propagation scenarios $[21,30]$ or to apply propagation models which do not take into account the probabilistic nature of all parameters (e.g., the number of paths) $[31,32]$. In the present paper, a completely probabilistic propagation model between each base station and each mobile is used which is characterized by the following properties.

The number of resolvable propagation paths is random and exhibits a binomial distribution (according to personal communication with U. Martin at Deutsche Telekom AG, 1999). Shadowing is modelled by a log-normal fading of the total received power [18, Section 3.1.1.2]. The random distribution of the total (log-normal fading) power to individual propagation paths (often denoted as macropaths or paths from scattering clusters) is modelled by applying an additional log-normal fading to the delayed paths with respect to the direct path (line of sight). Furthermore, a basic path attenuation and an extra attenuation that is proportional to the excess delay are taken into account. The basic attenuation is determined by the COST-Hata model [33] and a break point limits the attenuation to a certain minimum value for small distances. The excess delay of reflected paths is exponentially distributed leading to an exponential power delay profile [18, Section 3.1.1.3.3]. As mentioned 
TABLE 1: Simulation parameters.

\begin{tabular}{lc}
\hline Average number of mobiles per cell & 6 \\
Maximum number of mobiles per cell & 10 \\
Cell radius & $1 \mathrm{~km}$ \\
Carrier frequency & $2 \mathrm{GHz}$ \\
Antenna height of base stations & $30 \mathrm{~m}$ \\
Antenna height of mobile stations & $1.7 \mathrm{~m}$ \\
Break point that limits the attenuation at small distances & $100 \mathrm{~m}$ \\
Standard deviation of slow fading & $8 \mathrm{~dB}$ \\
Average number of paths & 3 \\
Maximum number of paths & 6 \\
Standard deviation of the attenuation of the delayed paths & $6 \mathrm{~dB}$ \\
with respect to the direct path & $8 \mathrm{~dB}$ \\
Average attenuation of the delayed paths with respect to the direct path & $4 \mathrm{~dB} / \mu \mathrm{s}$ \\
Additional attenuation proportional to the excess delay & $20^{\circ}$ \\
Standard deviation of the DoAs with respect to the direct path & $1^{\circ}$ \\
Standard deviation of the angular spread of each individual path &
\end{tabular}

TABle 2: Antenna arrays.

\begin{tabular}{lc}
\hline \multicolumn{2}{c}{ Circular antenna array } \\
$\begin{array}{lc}\text { Number of antenna elements } \\
\text { Radius of the array }\end{array}$ & $0.12 \mathrm{~m}$ \\
\hline \multicolumn{2}{c}{ Uniform linear array } \\
\hline $\begin{array}{lc}\text { Number of elements per sector } \\
\text { Number of sectors }\end{array}$ & 4 \\
Element spacing & $\lambda / 2=0.075 \mathrm{~m}$ \\
\hline
\end{tabular}

before, the directions of arrival which are denoted by $\hat{\theta}_{l, k, j(k)}$ obey a Laplacian distribution with respect to the direct path (standard deviation $=$ several tens of degrees) $[18$, Section 3.2.2.1]. Moreover, according to (28), the azimuthal power spectrum of each individual path is also incorporated in the simulations. As mentioned before, the azimuthal power spectra follow also a Laplacian shape (standard deviation in the order of one degree or less) and are identical for the different paths. In order to reduce the computational complexity, fast fading processes are included analytically as described in Section 4.

In the simulations, power control issues are completely neglected for downlink as well as uplink. The downlink transmit power values are assumed to be the same for all mobile stations, that is, $\check{P}_{k}=\check{P}$ for $k=0, \ldots, K-1$. It has to be mentioned that the capacity of the system increases when adopting power control since intracell interference is reduced. However, intercell interference is only marginally affected by power control. Finally, no handover issues are considered within this paper.

\subsection{Simulation methodology and parameters}

One main objective of this paper is to compare the performance gain for different smart antenna topologies. The key parameter to express performance is the outage probability for the given antenna concept. An outage occurs if the SIR of the mobile station after RAKE reception with maximum ratio combining falls below the service dependent required SIR threshold. Thus, the outage probability is given by the CDF of the SIR calculated versus all mobile stations in the reference cells. Since the SIR depends on the spreading gain and the spreading gain is determined by the specific service, we do not take into account the spreading gain. For all following numerical results, we set $G_{S}=1$. Note that the simulations are based on snapshots with fixed mobiles, where for each snapshot a CDF can be calculated. For each snapshot, we dice the locations of the mobiles as well as all other random variables. The following list gives a short overview of the main simulation steps.

(1) Based on the uplink transmission and using the reciprocity of uplink and downlink, we calculate the spatial covariances for downlink as well as the optimum beamforming weights.

(2) In a second step, the path diagrams are evaluated taking into account the beamformed diagram, the element specific diagrams, as well as the azimuthal power distribution of each resolvable path.

(3) With this, the user-specific SIRs after RAKE reception are known and can be used for CDF calculation.

(4) Finally, in order to compare the different array topologies, we average the CDF over all mobiles and over several snapshots, where different locations for the mobiles and different radio channels are determined. The averaged CDF allows to directly read the instantaneous outage probability of the downlink transmission.

The main simulation parameters are summarized in Tables 1 and 2. It has to be mentioned that for the system investigations we simulate $6 \cdot 7$ mobile stations within reference cells in average and 100 snapshots are carried out. Thus, the resulting $\mathrm{CDF}$ is calculated by averaging over $6 \cdot 7 \cdot 100=4200$ mobile stations. 


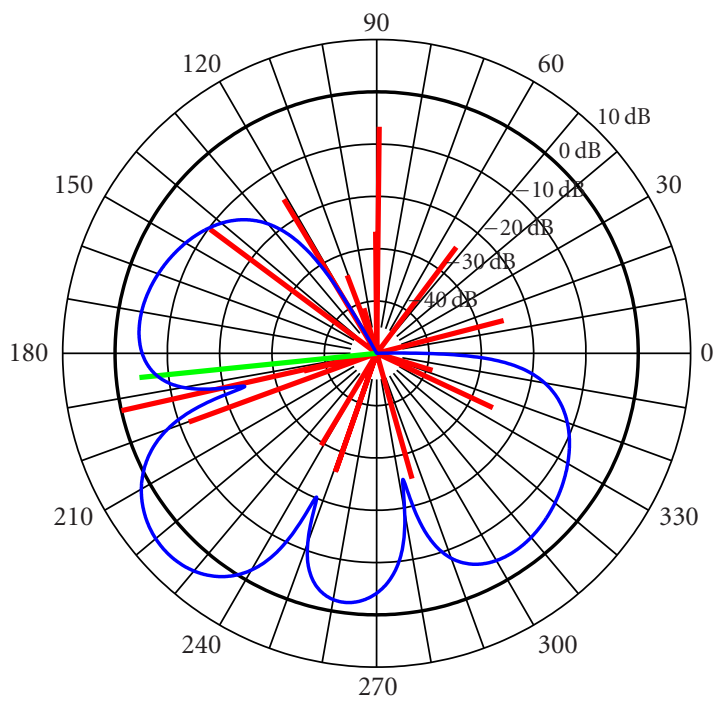

FIgURE 4: Example for an optimized path diagram in a sectorized system for a single sector (main beam direction is $240^{\circ}$ ). The ULA consists of 4 elements.

For illustration purposes, Figures 4 and 5 show examples of path diagrams for an identical propagation scenario. A system with three sectors and a ULA with 4 elements per sector (12 antenna elements in total) is compared with a system with circular arrays each of 12 elements. The bars in the diagrams correspond to the gain factors of the individual paths - for the displayed example only one desired path (at beam direction of $186^{\circ}$ ) exists.

Figure 4 shows the path diagram for the sectorized system. The backward attenuation of the antenna elements is $a_{\mathrm{R}}=60 \mathrm{~dB}$. It can be observed in the figure that the beamforming algorithm tries to suppress the undesired paths. Obviously, the four element antenna array does not exhibit sufficient degrees of freedom to generate all required nulls.

For the same propagation scenario, Figure 5 shows the optimization result for the circular array with 12 elements. Due to the larger number of antenna elements, the circular array is much more able to suppress the strong undesired paths.

\section{SIMULATION RESULTS}

\section{Overall performance comparison}

Figure 6 shows the different CDFs for the diverse antenna array topologies that are under investigation. The topologies we are interested in are as follows:

(a) one omnidirectional antenna per base station,

(b) three-sector base stations with one antenna element per sector and squared cosine characteristic,

(c) three-sector base stations where we apply one ULA with four elements per sector and squared cosine characteristic,

(d) one UCA with 12 omnidirectional antenna elements per base station.

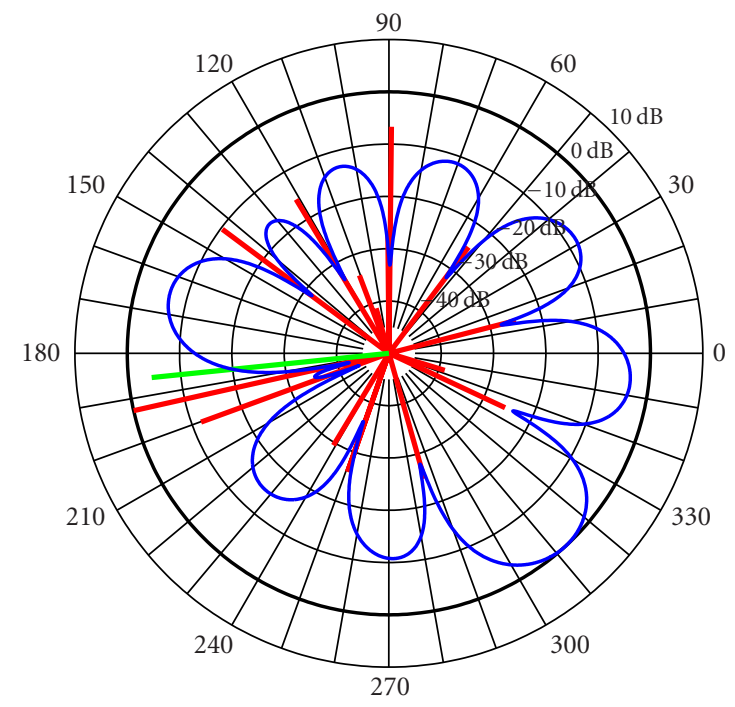

FIGURE 5: Example for an optimized path diagram for a circular antenna array with 12 omnidirectional elements.

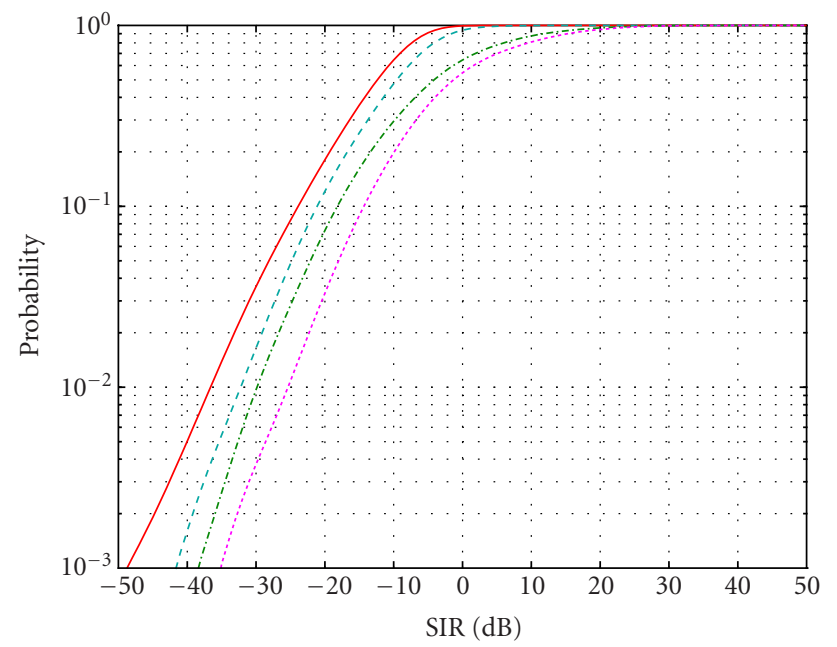
(a) Omnidirectional antennas
(b) Sectorization
(c) Sectorization with ULAs
(d) Circular arrays

FIGURE 6: Averaged CDF of the instantaneous SIR. Comparison between (a) reference system with omnidirectional antenna elements, (b) sectorized system with a single sector antenna per sector, (c) sectorized system with ULAs in each sector, four antenna elements per sector, and (d) system with circular antenna arrays and 12 omnidirectional antenna elements.

The omnidirectional topology is used as reference, while (b) is practically implemented today, and topologies (c) and (d) are under discussion for future implementation.

Figure 6 shows that for an outage probability of $10^{-2}$, simple sectorization yields a gain of about $4 \mathrm{~dB}$ compared to the omnidirectional configuration. The application of the linear array leads to an additional gain of about $3 \mathrm{~dB}$. The circular array is superior and indicates an extra gain of 


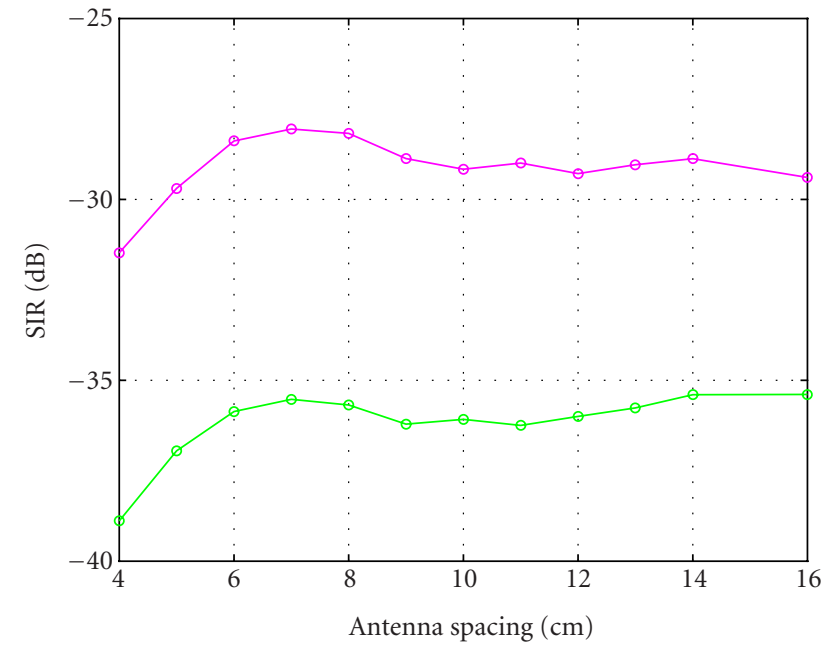

FIGURE 7: SIR for an outage probability of $10^{-2}$ versus ULA element spacing for sectorized system. Dark curve: 6 mobile stations per cell, light curve: 20 mobile stations per cell.

approximately $4 \mathrm{~dB}$ compared to the linear array topology. The latter gain can be explained as follows.

(i) The circular array is able to form narrower beams due to the larger number of antenna elements (4 per ULA compared to 12 per UCA). This means that nulls and maxima in the path diagram can be arranged more densely.

(ii) Due to the larger number of antenna elements, the circular array exhibits more nulls in the diagram. These nulls can be arranged more flexibly in order to perform nulling of the undesired and amplification of the desired paths. For example, if many strong undesired paths are located in a certain angular range, the circular array is more capable to suppress them while the ULA suffers due to its less powerful nulling capability in that range.

(iii) It is well known [15] that a ULA exhibits a low angular resolution for large angles (with respect to the main beam direction) while for the UCA this is not the case.

It has to be mentioned that the ULA performance is improved by handover between sectors of one base station (softer handover) [5]. But this technology is out of scope for this paper and might be an interesting task for future investigations.

\section{Spacing of antenna elements, backward attenuation, and half-power beamwidth}

An important parameter of an antenna array is the spacing of its elements. In the following, we discuss the impact of the antenna element spacing on the SIR. For the 3-sector system with ULAs, Figure 7 shows the SIR which is achieved for an average outage probability of $10^{-2}$ versus the antenna element spacing. We consider system loads of an average number of 6 and 20 mobile stations per cell, respectively. The higher the SIR for a given load the better the performance of

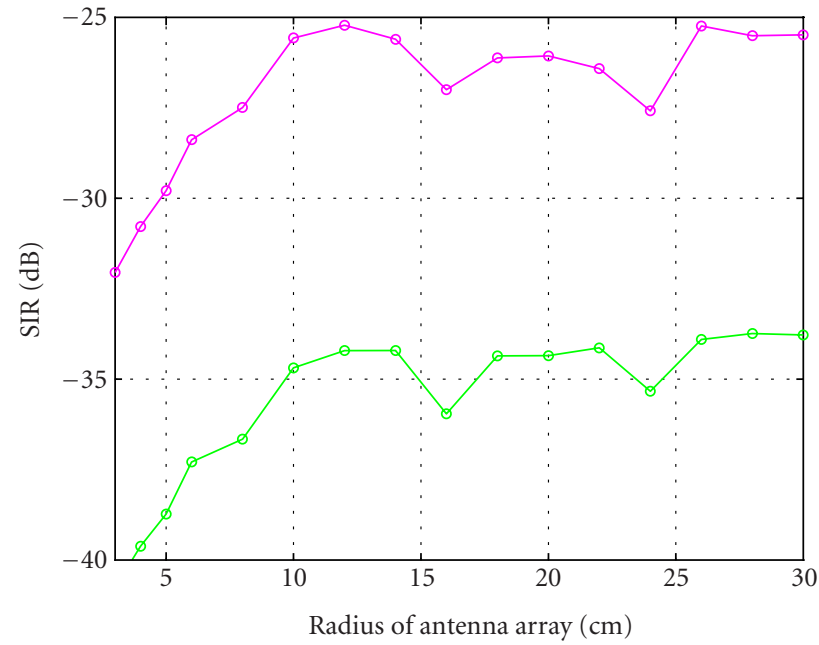

FIGURE 8: SIR for an outage probability of $10^{-2}$ versus circular array radius. Dark curve: 6 mobile stations per cell, light curve: 20 mobile stations per cell.

the antenna array, since the array is more capable to suppress the interference. We observe that the antenna spacing should be at least $\lambda / 2 \approx 7.5 \mathrm{~cm}$ independent of the given system load. For larger element spacing, the performance changes only slightly, while for small spacing it extremely degrades. The degradation can be explained by a reduced number of nulls in the path diagram for small antenna distances. A system with circular arrays is analyzed in Figure 8 . The radius of the circular array should be at least $12 \mathrm{~cm}$. This value corresponds to an antenna spacing of approximately $6.4 \mathrm{~cm}$ which is slightly less than $\lambda / 2$. Note that for all considered angular spread and spacings between the antenna elements, high correlation between antenna elements is still assumed. Figures 7 and 8 show curves for an average density of 6 and 20 mobiles per cell. It can be observed that the shape of the curves does not depend significantly on the average number of mobiles per cell.

From a practical perspective, antenna arrays with smaller dimensions are easier to adopt. Because of this aspect and because of the results of Figures 7 and 8, it can be concluded that half of the wavelength is the best suitable antenna spacing.

Next, Figure 9 shows the performance of a sectorized system (single antenna and ULA) for different backward attenuations of the antenna elements. No performance difference can be noticed between antenna elements with backward attenuations of 20 and $60 \mathrm{~dB}$. This result indicates that in sectorized systems, the requirements for the backward attenuation are less severe.

Up to here, we assumed a half power beamwidth $(3 \mathrm{~dB}$ angular aperture) of $120^{\circ}$ for sectorized systems. In the following, we study the impact of this design parameter on the system performance. Remember that we consider neither additive noise nor broadcast channels. Thus, the same maximum gain can be used for all antennas independently from the angular aperture. Corresponding to Figures 7 and 8, in 


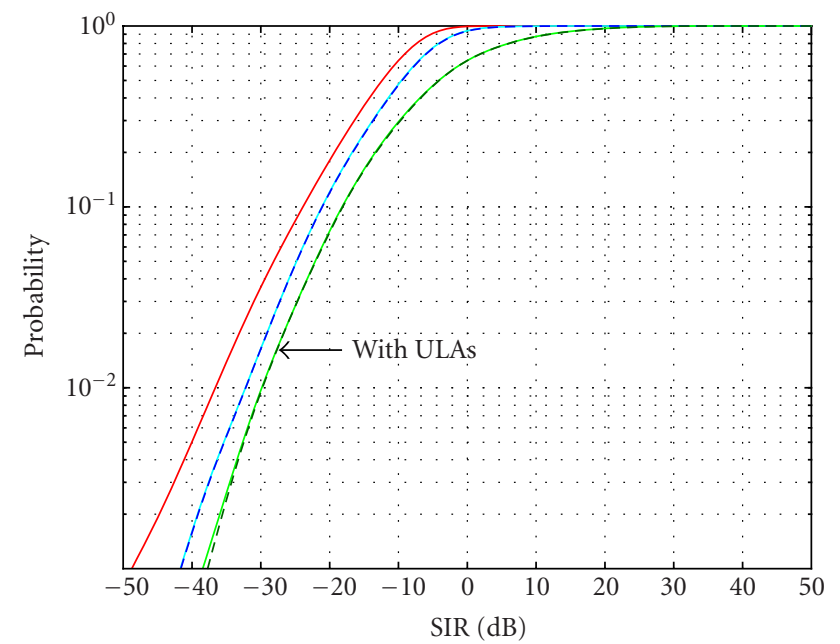

(a) Omnidirectional antennas
(b) Sectorization: $a_{\mathrm{R}}=20 \mathrm{~dB}$
(c) Sectorization with ULAs: $a_{\mathrm{R}}=20 \mathrm{~dB}$
--- (d) Sectorization: $a_{\mathrm{R}}=60 \mathrm{~dB}$
--- (e) Sectorization with ULAs: $a_{\mathrm{R}}=60 \mathrm{~dB}$

FIgURE 9: Averaged CDF of the instantaneous SIR. Comparison between (a) reference system with omnidirectional antenna elements, (b) sectorized system with a single sector antenna per sector $\left(a_{\mathrm{R}}=20 \mathrm{~dB}\right),(\mathrm{c})$ sectorized system with ULAs in each sector, four sector antennas per sector $\left(a_{\mathrm{R}}=20 \mathrm{~dB}\right),(\mathrm{d})$ sectorized system with a single sector antenna per sector $\left(a_{\mathrm{R}}=60 \mathrm{~dB}\right)$, (e) sectorized system with ULAs in each sector, four sector antennas per sector $\left(a_{\mathrm{R}}=60 \mathrm{~dB}\right)$.

Figure 10 the SIR for an outage probability of $10^{-2}$ is shown versus the half-power beamwidth. It can be seen that an angular aperture of $120^{\circ}$ is not optimum. The optimum value is of about $150^{\circ}$. But, the optimum reveals to be very wide leading to almost no performance degradation if the angular aperture is in the range $120^{\circ}-220^{\circ}$.

\section{Circular array with sector elements}

In our final investigations, we analyze the system performance of a circular array when sector antenna elements are applied instead of elements with omnidirectional antenna patterns. The beam of each antenna element is pointing in radial direction (see Figure 11). Such an antenna array models an array that surrounds an inner mast where the shadowing of the antenna mast cannot be neglected. For simplicity, antenna diagrams described by (27) are applied. Figure 12 depicts the SIR for a given outage probability of $10^{-2}$ versus the $3 \mathrm{~dB}$ beamwidth of the sector antennas. For a 12 element circular array it can be observed that already for small beamwidths of about $40^{\circ}$ the optimum performance of omnidirectional antennas is achieved.

The importance of adopting sector antennas in circular antenna arrays has to be emphasized since because of the mutual coupling between antenna elements and even without a mast in the center, it is difficult to develop circular antenna arrays with omnidirectional antenna patterns. An additional

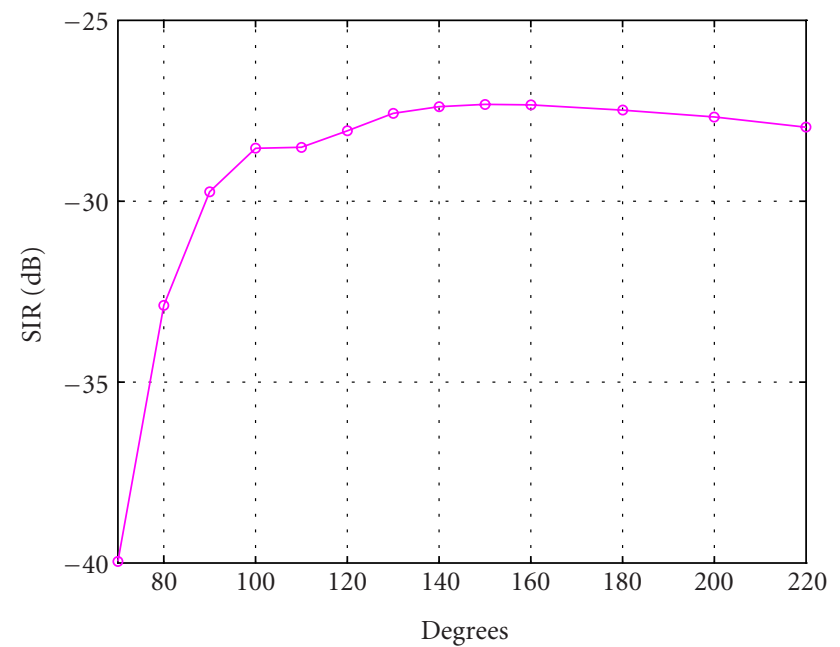

FIGURE 10: SIR for an outage probability of $10^{-2}$ versus $3 \mathrm{~dB}$ beamwidth of sector antennas.

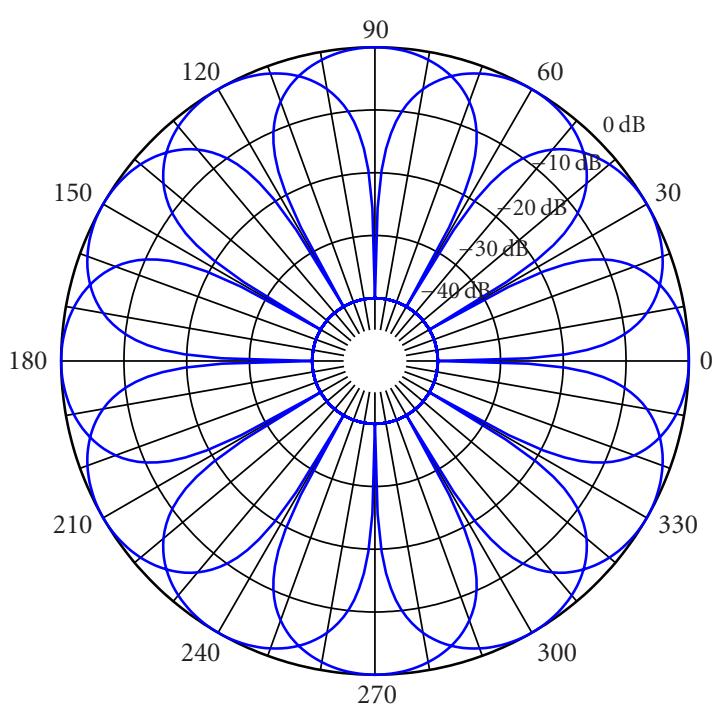

Figure 11: Antenna diagrams of all elements of a 12 element antenna array with a beamwidth of $30^{\circ}$. Backward attenuation $a_{\mathrm{R}}=$ $40 \mathrm{~dB}$.

advantage of using sector antennas is that their mutual coupling is weaker than between omnidirectional antenna elements.

\section{CONCLUSION}

A cellular model for system level investigations of antenna arrays has been presented. A new simulation methodology has been applied, which takes into account the gain of path diversity in a realistic manner. With the described assumptions and approximations it was possible to determine upper limits for the SIR gain when smart antennas are applied in CDMA-based mobile radio networks.

The CDF (outage probability) of the SIR after RAKE reception with maximum ratio combing is compared for 


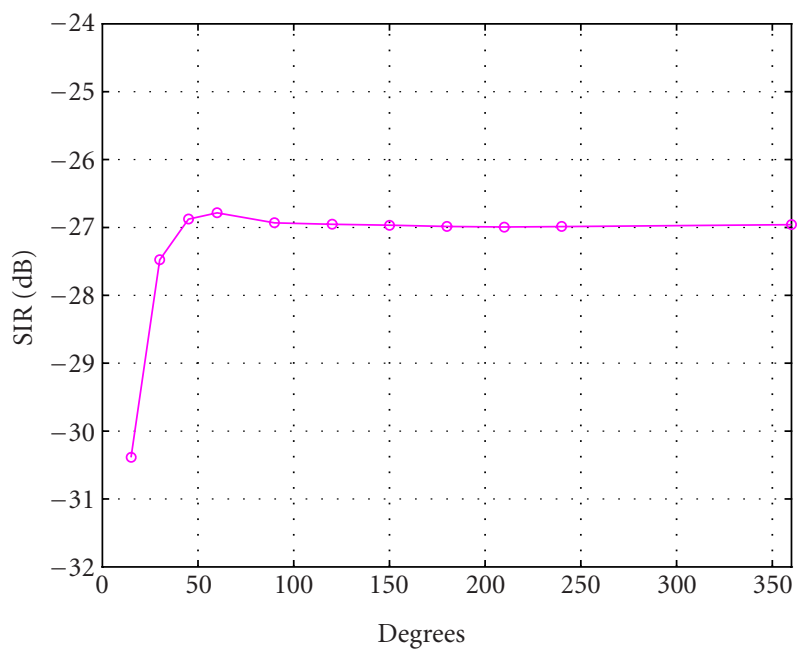

FIGURE 12: SIR for an outage probability of $10^{-2}$ versus $3 \mathrm{~dB}$ beamwidth of sector antennas of a system with circular antenna arrays. For comparison, the beamwidth of $360^{\circ}$ corresponds to omnidirectional antenna elements.

networks with and without sectorization, as well as with and without smart antenna arrays. For a fair comparison of diverse smart antenna array topologies, we considered networks with the same number of antenna elements at each base station. The lowest outage probability was found for networks applying circular antenna arrays. The gain with respect to the 3-sector system with one ULA per sector is of about $4 \mathrm{~dB}$.

Furthermore, the parameters of the antenna arrays have been optimized by extensive simulations. The observed results indicate that the element spacing should be approximately half of the wavelength-independently from the antenna topology. Only slight performance changes have been observed for larger element spacings, while for small element distances the performance degrades.

Concerning the backward attenuation of the elementspecific antenna diagrams, the results show that the backward attenuation can be as low as $20 \mathrm{~dB}$ without any performance degradation. Furthermore, the $3 \mathrm{~dB}$ beamwidth is also an uncritical parameter-it may be within the range $120^{\circ}-$ $220^{\circ}$.

Finally, no performance degradation has been observed for circular arrays if sector antennas with reasonably large beamwidths are used instead of omnidirectional antenna elements.

\section{REFERENCES}

[1] E. Dahlman, B. Gudmundson, M. Nilsson, and A. Sköld, "UMTS/IMT-2000 based on wideband CDMA," IEEE Communications Magazine, vol. 36, no. 9, pp. 70-80, 1998.

[2] T. Ojanperä and R. Prasad, "An overview of air interface multiple access for IMT-2000/UMTS," IEEE Communications Magazine, vol. 36, no. 6, pp. 82-95, 1998.

[3] H. Boche and M. Schubert, "Theoretical and experimental comparison of optimization criteria for downlink beamform- ing," European Transactions on Telecommunications, vol. 12, no. 5, pp. 417-426, 2001, Special Issue on Smart Antennas.

[4] R. M. Buehrer, A. G. Kogiantis, S.-C. Liu, J. Tsai, and D. Uptegrove, "Intelligent antennas for wireless communications uplink," Bell Labs Technical Journal, vol. 4, no. 3, pp. 73-103, 1999.

[5] H. Holma and A. Toskala, WCDMA for UMTS, John Wiley \& Sons, Chichester, UK, 1st edition, 2000.

[6] A. Yener, R. D. Yates, and S. Ulukus, "Interference management for CDMA systems through power control, multiuser detection, and beamforming," IEEE Trans. Communications, vol. 49, no. 7, pp. 1227-1239, 2001.

[7] J. Muckenheim and U. Bernhard, "A framework for load control in 3rd generation CDMA networks," in Proc. IEEE Global Telecommunications Conference, vol. 6, pp. 3738-3742, San Antonio, Tex, USA, November 2001.

[8] J.-W. Liang and A. J. Paulraj, "On optimizing base station antenna array topology for coverage extension in cellular radio networks," in Proc. IEEE 45th Vehicular Technology Conference, vol. 2, pp. 866-870, Chicago, Ill, USA, July 1995.

[9] J. Fuhl, D. J. Cichon, and E. Bonek, "Optimum antenna topologies and adaptation strategies for SDMA," in Proc. IEEE Global Telecommunications Conference, vol. 1, pp. 575-580, London, UK, November 1996.

[10] R. Martinez, D. Trosa, L. de Haro, and M. Calvo, "Smart antennas performance evaluation and capacity increase for WCDMA UMTS," in Proc. IEEE 53rd Vehicular Technology Conference, vol. 1, pp. 147-151, Rhodes, Greece, May 2001.

[11] A. Osseiran, M. Ericson, J. Barta, B. Goransson, and B. Hagerman, "Downlink capacity comparison between different smart antenna concepts in a mixed service W-CDMA system," in Proc. IEEE 54th Vehicular Technology Conference, vol. 3, pp. 1528-1532, Atlantic City, NJ, USA, October 2001.

[12] M. Pettersen, L. E. Braten, and A. G. Spilling, "An evaluation of adaptive antennas for UMTS FDD by system simulations," in Proc. IEEE Vehicular Technology Conference, vol. 1, pp. 227231, Orlando, Fla, USA, October 2003.

[13] A. Czylwik and A. Dekorsy, "System level simulations for downlink beamforming with different array topologies," in Proc. IEEE Global Telecommunications Conference, vol. 5, pp. 3222-3226, San Antonio, Tex, USA, November 2001.

[14] A. Czylwik and A. Dekorsy, "Optimization of downlink beamforming for systems with frequency division duplex," in Proc. IEEE International Zurich Seminar on Broadband Communications, pp. 11-1-11-6, Zurich, Switzerland, February 2002.

[15] J. Litva and T. K.-Y. Lo, Digital Beamforming in Wireless Communications, Artech House, Boston, Mass, USA, 1st edition, 1996.

[16] M. Schacht, A. Dekorsy, and P. Jung, "Downlink beamforming concepts in UTRA FDD," in Kleinheubacher Tagung, Kleinheubach, Germany, September 2002.

[17] K. I. Pedersen, P. E. Mogensen, and F. Frederiksen, "Jointdirectional properties of uplink and downlink channel in mobile communications," Electronics Letters, vol. 35, no. 16, pp. 1311-1312, 1999.

[18] L. M. Correia, Wireless Flexible Personalised Communications: COST 259: European Co-operation in Mobile Radio Research, John Wiley \& Sons, Chichester, UK, 2001.

[19] K. Hugl, K. Kalliola, and J. Laurila, "Spatial reciprocity of uplink and downlink radio channels in FDD systems," in Proc. COST 273 Technical Document TD(02)066, p. 7, Espoo, Finland, May 2002.

[20] M. Haardt, Efficient one-, two-, and multidimensional highresolution array signal processing, $\mathrm{Ph} . \mathrm{D}$. thesis, Lehrstuhl für Netzwerktheorie und Schaltungstechnik, Technische Universität, Munich, Germany, 1997. 
[21] W. Utschick and J. A. Nossek, "Downlink beamforming for FDD mobile radio systems based on spatial covariances," in Proc. European Wireless 99 \& ITG Mobile Communications, pp. 65-67, Munich, Germany, October 1999.

[22] D. Filho, C. Panazio, F. Cavalcanti, and J. Romano, "On downlink beamforming techniques for TDMA/FDD systems," in Proc. 19th Symposium on Brazilian Telecommunications, Fortaleza-CE, Brazil, 2001.

[23] B. Chalise, L. Häring, and A. Czylwik, "Robust UL to DL spatial covariance matrix transformation for DL beamforming," in Proc. IEEE Global Telecommunications Conference (GLOBECOM '03), San Francisco, Calif, USA, December 2003.

[24] A. Czylwik, "Downlink beamforming for mobile radio systems with frequency division duplex," in Proc. IEEE 11th International Symposium on Personal, Indoor and Mobile Radio Communications, vol. 1, pp. 72-76, London, UK, September 2000.

[25] A. Czylwik, "Comparison and optimization of antenna concepts for downlink beamforming," in Proc. International Conference on Telecommunications, Papeete, French Polynesia, France, 2003.

[26] J. G. Proakis, Digital Communications, McGraw-Hill, New York, NY, USA, 3rd edition, 1995.

[27] F. Rashid-Farrokhi, K. J. R. Liu, and L. Tassiulas, "Transmit beamforming and power control for cellular wireless systems," IEEE Journal on Selected Areas in Communications, vol. 16, no. 8, pp. 1437-1450, 1998.

[28] M. Schubert and H. Boche, "A unifying theory for uplink and downlink multi-user beamforming," in Proc. International Zurich Seminar on Broadband Communications, pp. 271-276, Zurich, Switzerland, February 2002.

[29] E. Visotsky and U. Madhow, "Optimum beamforming using transmit antenna arrays," in Proc. IEEE 49th Vehicular Technology Conference, vol. 1, pp. 851-856, Houston, Tex, USA, July 1999.

[30] C. Farsakh and J. A. Nossek, "Spatial covariance based downlink beamforming in an SDMA mobile radio system," IEEE Trans. Communications, vol. 46, no. 11, pp. 1497-1506, 1998.

[31] G. G. Raleigh, S. N. Diggavi, V. K. Jones, and A. Paulraj, "A blind adaptive transmit antenna algorithm for wireless communication," in Proc. IEEE International Conference on Communications, vol. 3, pp. 1494-1499, Seattle, Wash, USA, June 1995.

[32] H. Asakura and T. Matsumoto, "Cooperative signal reception and down-link beam forming in cellular mobile communications," IEEE Trans. Vehicular Technology, vol. 48, no. 2, pp. 333-341, 1999.

[33] J. S. Lee and L. E. Miller, CDMA Systems Engineering Handbook, Artech House, Boston, Mass, USA, 1st edition, 1998.

Andreas Czylwik studied electrical engineering at the Technical University of Darmstadt, Germany, from 1978 to 1983. In 1988, he received his Dr.-Ing. degree and in 1994 his Habilitation degree, both from the Technical University of Darmstadt and both in the field of optical communications. From 1994 to 2000, he was with the research and development center (Technologiezentrum) of Deutsche Telekom in the Depart-

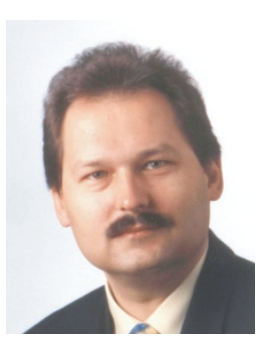
ment of Local Area Broadband Radio Systems. He was in charge of several research projects, for example, a broadband radio communication demonstrator based on single carrier transmission with frequency domain equalization, as well as several projects on smart antenna concepts in cellular mobile radio systems. In 2000, he became a full professor at the Technical University of Braunschweig, heading the Department of Microcellular Radio Systems. Since 2002, he has been with the University Duisburg-Essen and in charge of the Department of Communication Systems. He was an Editor for the IEEE Journal on Selected Areas in Communications and IEEE Transactions on Wireless Communications. His research interests are in the field of adaptive transmission techniques in radio communications, such as smart antennas and adaptive modulation and coding techniques.

Armin Dekorsy received his Dipl.-Ing. (FH) (B.S.) degree from Fachhochschule Konstanz, Germany, 1992, his Dipl.-Ing. (M.S.) degree from University of Paderborn, Germany, 1995, and his Ph.D. degree from University of Bremen, Bremen, Germany, 2000, all in electrical engineering. From 2000 to 2001 he was with T-Nova Deutsche Telekom Innovationsgesellschaft $\mathrm{mbH}$, Darmstadt, Germany, where he was

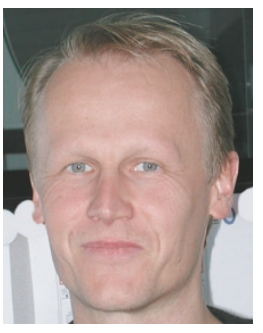
leading research projects on smart antenna technologies. In 2001, he joined Lucent Technologies Network Systems GmbH, Nuremberg, Germany. Since October 2003 he has been with Bell Labs Advanced Technologies and is currently conducting research projects on radio resource management algorithms including interference cancellation techniques. He also contributes to marketing strategies, manages government funded research projects, and presents the Bell Labs Advanced Technologies at numerous seminars. His current research interests are mainly smart antenna solutions, interference cancellation techniques, as well as radio resource management algorithms for third-generation mobile standards. 